\title{
NÍVEIS DE NITROGÊNIO POR FERTIRRIGAÇÃO E DENSIDADE DE PLANTIO NA CULTURA DO MELÃO EM UM VERTISSOLO ${ }^{1}$
}

\author{
CLEMENTINO MARCOS BATISTA DE FARIA², NIVALDO DUARTE COSTA ${ }^{2}$, \\ JOSÉ MARIA PINTO ${ }^{2}$, LUIZA TEIXEIRA DE LIMA BRITO ${ }^{3}$ e JOSÉ MONTEIRO SOARES ${ }^{2}$
}

\begin{abstract}
RESUMO - O trabalho constou de um experimento com melão (Cucumis melo L.), conduzido em um Vertissolo, em Juazeiro, BA, em 1995, com o objetivo de avaliar o efeito de níveis de N por fertirrigação e de densidades de plantio na produtividade e qualidade de fruto. Os níveis de $\mathrm{N}$ foram $0,80,130$ e $180 \mathrm{~kg} / \mathrm{ha}$, combinados com os espaçamentos 2,00 e 1,80 m entre linhas e 0,20 m entre plantas, com uma ou duas plantas por cova. A fonte de $\mathrm{N}$ foi a uréia, aplicada diariamente até 42 dias após a germinação, por meio da irrigação por gotejamento. Todos os tratamentos receberam uma adubação uniforme de $120 \mathrm{~kg} / \mathrm{ha}$ de $\mathrm{P}_{2} \mathrm{O}_{5}$ e $120 \mathrm{~kg} / \mathrm{ha}$ de $\mathrm{K}_{2} \mathrm{O}$. Os espaçamentos entre linhas não causaram diferenças significativas em nenhuma variável estudada. O nível de $80 \mathrm{~kg} / \mathrm{ha}$ de $\mathrm{N}$ combinado com uma planta por cova proporcionou uma produtividade de 34,07 t/ha, com 55,7\% de frutos próprios para o mercado interno, não-significativamente $(\mathrm{P} \leq 0,05)$ inferior à produtividade obtida com os níveis mais elevados de $\mathrm{N}$ em qualquer combinação. Com este mesmo nível, obtiveram-se frutos com $10,22^{\circ}$ Brix significativamente $(\mathrm{P} \leq 0,05)$ superior ao do tratamento sem $\mathrm{N}$ e não-significativamente inferior ao dos outros níveis. Para se obter uma maior parte de frutos próprios para o mercado externo, foi necessário elevar a densidade para duas plantas por cova e o nível de $\mathrm{N}$ para $130 \mathrm{ou} 180 \mathrm{~kg} / \mathrm{ha}$. O peso médio dos frutos aumentou de 1,008 para $1,705 \mathrm{~kg}$, à medida que foram aumentados os níveis de $\mathrm{N}$ ou se diminuiu a densidade de plantio de duas para uma planta por cova.
\end{abstract}

Termos para indexação: Cucumis melo, qualidade dos frutos, tamanho dos frutos, Brix.

\section{NITROGEN LEVELS THROUGH FERTIRRIGATION AND PLANT DENSITY ON MELON CROP IN A VERTISOL}

\begin{abstract}
This study consisted of one experiment with melon (Cucumis melo L.), carried out in a Vertisol in Juazeiro, BA, Brazil, in 1995, with the objective of evaluating the effects of nitrogen levels through fertirrigation and plant density on fruit yield and quality. The $\mathrm{N}$ levels were $0,80,130$ and $180 \mathrm{~kg} / \mathrm{ha}$, combined with row spacings of 2.0 and $1.8 \mathrm{~m}$ and $0.20 \mathrm{~m}$ between plants within the row, with one or two plants/hole. The source of $\mathrm{N}$ was urea applied daily up to 42 days after germination, through drip irrigation. All treatments had a uniform fertilization of $120 \mathrm{~kg} / \mathrm{ha}$ of $\mathrm{P}_{2} \mathrm{O}_{5}$ and $120 \mathrm{~kg} / \mathrm{ha}$ of $\mathrm{K}_{2} \mathrm{O}$. No significant difference was caused by spacing between rows in the studied variables. Eighty $\mathrm{kg} / \mathrm{ha}$ of $\mathrm{N}$ combined with one plant per hole gave a yield of $34.07 \mathrm{ton} / \mathrm{ha}$, being $55,7 \%$ of fruit allocated to inside market, not significantly lower than those obtained with the highest $\mathrm{N}$ levels in any combination. This same $\mathrm{N}$ level gave fruits with $10.22^{\circ}$ Brix, significantly higher than the treatment without $\mathrm{N}$ and not significantly lower than the other levels. In order to get high number of good fruit for out side market it was necessary to elevate the density to two plants per hole and $\mathrm{N}$ level to 130 or $180 \mathrm{~kg} / \mathrm{ha}$. Mean weight of fruits increased from 1.008 to $1.705 \mathrm{~kg}$ with the increase in the levels of $\mathrm{N}$ and the decrease in plant density of two plants per hole to one plant per hole.
\end{abstract}

Index terms: Cucumis melo, fruit quality, fruit size, Brix.

\footnotetext{
${ }^{1}$ Aceito para publicação em 30 de março de 1999.

Apresentado no XIII Congresso Latino-Americano de Ciência do Solo, com auxílio financeiro da FACEP. Financiado pela Embrapa/Petrobras.

${ }^{2}$ Eng. Agrôn., M.Sc., Embrapa-Centro de Pesquisa Agropecuária do Trópico Semi-Árido (CPATSA), Caixa Postal 23, CEP 56300-000 Petrolina, PE.

${ }^{3}$ Eng. Agríc., M.Sc., Embrapa-CPATSA.
}

\section{INTRODUÇÃO}

A cultura do melão exige nível tecnológico adequado, pela necessidade de se obterem frutos de boa qualidade para atender às exigências dos mercados interno e externo, com relação ao tamanho e formato do fruto, à cor da casca e ao Brix da polpa. No Submédio São Francisco, a 
comercialização é bem aceita quando os frutos são dos tamanhos 6 e 8 para o mercado interno e 10 e 12 para o mercado externo, e têm formato arredondado ou ligeiramente oval, cor amarela, e um Brix superior a 10 graus. No entanto, as pesquisas com esta cucurbitácea ainda são bastante restritas, no Brasil, principalmente no Nordeste.

Em relação à adubação, Srinivas \& Prabhakar (1984) obtiveram aumento de $200 \%$ na produtividade de melão com a aplicação de 50 kg/ha de N. Wilcox (1973) observou que as maiores produtividades de melão foram obtidas com os níveis de 80 a $90 \mathrm{~kg} / \mathrm{ha}$ de N. Das várias doses de N testadas por Meisheri et al. (1984) na cultura do melão, $80 \mathrm{~kg} / \mathrm{ha}$ foi considerada a melhor. No trabalho de revisão de literatura realizado por Faria (1990), a média das doses adequadas de $\mathrm{N}$ obtidas em pesquisas ou recomendadas para adubação do melão é de $76 \mathrm{~kg} / \mathrm{ha}$ de N. Na região do Submédio São Francisco, Faria et al. (1994) encontraram que o nível econômico de $\mathrm{N}$ foi de $74 \mathrm{~kg} / \mathrm{ha}$ de $\mathrm{N}$ para o melão irrigado num Vertissolo. Bhella \& Wilcox (1986) observaram que a aplicação de $67 \mathrm{~kg} / \mathrm{ha}$ de $\mathrm{N}$ ao solo mais 50 ou $100 \mathrm{mg} / \mathrm{L}$ de $\mathrm{N}$ na água de irrigação por gotejamento, proporcionou a produtividade mais alta do melão.

Além da produtividade, o $\mathrm{N}$ também exerce efeito benéfico na qualidade dos frutos do melão, aumentando o número, peso e Brix, conforme Srinivas \& Prabhakar (1984), Prabhakar et al. (1985) e Faria et al. (1994), como também, melhorando a consistência da polpa, o formato e a cor dos frutos (Bhella \& Wilcox,1986).

A adubação via água de irrigação, denominada de fertirrigação, é hoje de comprovada eficácia no aumento de produtividade, na melhoria da qualidade dos frutos, na redução de mão-de-obra, do consumo de energia e dos gastos com equipamentos, e na maior eficiência na utilização de nutrientes (Costa et al., 1986), principalmente os mais móveis, como o N.

No tocante à densidade de plantio, as pressões exercidas pela população de plantas afetam de modo marcante o seu desenvolvimento. Quando a densidade de plantas aumenta por unidade de área, atinge-se um ponto no qual as plantas competem por fatores essenciais de crescimento, como nutrientes, luz e água (Janick, 1968). O espaçamento entre plantas ou a densidade de plantio tem influência no número e tamanho de frutos do meloeiro. O emprego de espaçamento adequado proporciona alta eficiência produtiva do cultivo.

Farias (1988) constatou que aumentando os espaçamentos entre plantas de melão de $0,30,0,45$, 0,60 até $0,75 \mathrm{~m}$ em fileiras com espaçamento de $0,80 \mathrm{~m}$, aumentou o peso médio dos frutos e a porcentagem de plantas produtivas. Paris et al. (1988), trabalhando com melão na Flórida, com densidades de 10, 20 e 30 mil plantas por hectare, e em Israel, com 5, 10, 20 e 40 mil plantas por hectare, verificaram que com o aumento da densidade de plantio na Flórida o rendimento não foi significativamente afetado, mas em Israel o número de frutos e a produtividade aumentaram significativamente, e o tamanho dos frutos diminuiu em ambos locais.

O espaçamento varia em função do nível de tecnologia empregado pelo produtor. No Submédio São Francisco, os espaçamentos mais comuns adotados pelos produtores são $2,0 \mathrm{~m}$ ou 1,8 $\mathrm{m}$ entre as filas e $0,5 \mathrm{~m}$ até $1,5 \mathrm{~m}$ entre plantas, geralmente, com uma planta por cova, ou com duas, quando a distância entre as covas é maior. As empresas com alto nível tecnológico têm adotado espaçamentos de 2,0 a $3,0 \mathrm{~m}$ entre fileiras e 0,3 a $0,8 \mathrm{~m}$ entre plantas. No caso de produção voltada para exportação, é possível fazer o plantio em fileiras duplas, deixando-se uma planta em cada lado do gotejador ou do sulco de irrigação. Isso permite intensa competição entre plantas que produzem maior número de frutos, de tamanho menor.

O objetivo deste trabalho foi estabelecer doses de $\mathrm{N}$ e densidade de plantio adequados para o cultivo do meloeiro com fertirrigação, no Submédio São Francisco, visando a maiores produtividade e qualidade dos frutos para os mercados interno e externo.

\section{MATERIAL E MÉTODOS}

O trabalho constou de um experimento com a cultura do melão (Cucumis melo L.), cultivar Eldorado 300, conduzido no Campo Experimental de Mandacaru, da Embrapa-Centro de Pesquisa Agropecuária do Trópico Semi-Árido (CPATSA), em Juazeiro, BA, no período de 
setembro a dezembro de 1995, em um Vertissolo com as seguintes características: areia $=16 \% ; \mathrm{pH}\left(\mathrm{H}_{2} \mathrm{O}\right)=7,0$; $\mathrm{Ca}^{2+}=24,4 \mathrm{cmol}_{\mathrm{C}} / \mathrm{dm}^{3} ; \mathrm{Mg}^{2+}=7,6 \mathrm{cmol}_{\mathrm{c}} / \mathrm{dm}^{3} ; \mathrm{Na}^{+}=$ $0,20 \mathrm{cmol}_{\mathrm{c}} / \mathrm{dm}^{3} ; \mathrm{K}^{+}=0,48 \mathrm{cmol}_{\mathcal{C}} / \mathrm{dm}^{3} ; \mathrm{Al}^{3+}=0,00 \mathrm{cmol}_{\mathrm{d}} / \mathrm{dm}^{3}$; e P $($ Bray 1$)=5 \mathrm{mg} / \mathrm{L}$, determinadas segundo metodologia da Embrapa (1979).

O delineamento experimental foi em blocos casualizados em faixa, com quatro repetições, e 16 tratamentos, constituídos de três fatores: níveis de $\mathrm{N}$, espaçamento entre filas e número de plantas por covas combinados entre si. Os níveis de $\mathrm{N}$ foram: $0,80,130$ e $180 \mathrm{~kg} / \mathrm{ha}$; os espaçamentos foram 2,00 m e 1,80 m, e o número de plantas por cova foi de uma e duas, com espaços, entre si, de $0,20 \mathrm{~m}$. Todos os tratamentos receberam adubação uniforme com $120 \mathrm{~kg} / \mathrm{ha}$ de $\mathrm{P}_{2} \mathrm{O}_{5}$ e $120 \mathrm{~kg} / \mathrm{ha}$ de $\mathrm{K}_{2} \mathrm{O}$. As parcelas tinham a área de 36 ou $40 \mathrm{~m}^{2}$, conforme o espaçamento entre filas.

Adotou-se o sistema de irrigação por gotejamento em linha, com gotejadores a espaços de $1,0 \mathrm{~m}$, vazão de $4 \mathrm{~L} / \mathrm{h}$ e pressão de $10 \mathrm{~m}$ de coluna de água. As irrigações foram feitas diariamente, com base na evaporação de água do tanque classe A e no coeficiente de cultura $(\mathrm{Kc})$. Todo o P foi aplicado em fundação, e o $\mathrm{N}$ e o K foram aplicados diariamente, até 42 dias após a emergência das plantas, via água de irrigação, utilizando-se um injetor hidráulico de fertilizantes. Os fertilizantes utilizados foram uréia, superfosfato simples e cloreto de potássio. Foram realizadas duas colheitas no experimento e avaliados os dados de produtividade, peso médio de frutos, Brix e classificação de frutos por tipo, ou seja, tamanho, que indica o número de frutos por caixa de embalagem, com as seguintes dimensões: $54 \mathrm{~cm}$ de comprimento, $34 \mathrm{~cm}$ de largura e $17 \mathrm{~cm}$ de altura. Exemplo, melão tipo 6, significa que a caixa contém seis melões.

\section{RESULTADOS E DISCUSSÃO}

Houve efeito significativo dos níveis de $\mathrm{N}$ e do número de plantas por cova e da interação desses fatores na produtividade e peso médio de frutos e, ainda, dos níveis de $\mathrm{N}$ no Brix dos frutos. Os espaçamentos entre filas não causaram diferenças significativas em nenhuma variável, provavelmente porque a diferença entre eles era pequena. Os efeitos dos níveis de $\mathrm{N}$ foram lineares, porém com ajuste baixo das equações $\left(r^{2}=0,62\right.$ com uma planta por cova e $\mathrm{r}^{2}=0,40$ com duas plantas por cova); por essa razão, usou-se o teste de médias de Duncan a $5 \%$ de probabilidade, para fazer avaliação dos resultados. A maior produtividade (37,71 t/ha) ocorreu no nível $180 \mathrm{~kg} / \mathrm{ha}$ de $\mathrm{N}$ com uma planta por cova, e a menor (20,20 t/ha), no nível zero de $\mathrm{N}$ com duas plantas por cova (Tabela 1). Observa-se, ainda, uma produtividade também alta (34,07 t/ha), não significativamente inferior à mais alta, e obtida no nível de $80 \mathrm{~kg} / \mathrm{ha}$ de $\mathrm{N}$ com uma planta por cova, que é um tratamento com menos custos. Esse nível assemelha-se aos níveis ótimos, encontrados por Wilcox (1973), Meisheri et al. (1984) e Faria et al. (1994), que foram de 80 a 90, 80 e $74 \mathrm{~kg} / \mathrm{ha}$, respectivamente.

As produtividades com $0 \mathrm{~kg} / \mathrm{ha}$ de $\mathrm{N}$ foram sempre inferiores às obtidas com a aplicação de $\mathrm{N}$, como era esperado, considerando a pobreza dos solos da região semi-árida nesse elemento (Black, 1968).

De modo geral, as produtividades com duas plantas por cova foram inferiores às obtidas com uma planta por cova, em virtude da concorrência acirrada entre as plantas e a saturação de massa verde na área.

Com duas plantas por cova, foi o único caso em que a produtividade aumentou entre os níveis 80 e $180 \mathrm{~kg} / \mathrm{ha}$ de $\mathrm{N}$ e pouco se alterou entre os níveis 130 e $180 \mathrm{~kg} / \mathrm{ha}$ de $\mathrm{N}$, enquanto com uma planta por cova houve diferença significativa na produtividade entre os níveis 130 e $180 \mathrm{~kg} / \mathrm{ha}$ de $\mathrm{N}$ (Tabela 1). Esse comportamento diferenciado explica a existência da interação entre os dois fatores.

Do mesmo modo, os dados do peso médio de frutos do melão aumentaram de valores, de uma

TABELA 1. Produtividade do melão em função dos níveis de $\mathrm{N}$ e do número de plantas por cova, Juazeiro, BA, 19951.

\begin{tabular}{ccc}
\hline \multirow{2}{*}{$\begin{array}{c}\text { Nitrogênio } \\
(\mathrm{kg} / \mathrm{ha})\end{array}$} & \multicolumn{2}{c}{ Número de plantas por cova } \\
\cline { 2 - 3 } & \multicolumn{1}{c}{1} & \multicolumn{1}{c}{2} \\
\hline & $-------------(\mathrm{t} / \mathrm{ha})$ & ------------- \\
0 & $25,14 \mathrm{cA}$ & $20,20 \mathrm{cA}$ \\
80 & $34,07 \mathrm{abA}$ & $25,36 \mathrm{aB}$ \\
130 & $29,98 \mathrm{bA}$ & $27,84 \mathrm{abA}$ \\
180 & $37,71 \mathrm{aA}$ & $30,00 \mathrm{aB}$ \\
\hline
\end{tabular}

${ }^{1}$ Médias seguidas pela mesma letra, minúscula nas colunas e maiúscula nas linhas, não diferem pelo teste de Duncan a $5 \%$ de probabilidade; F/Nitrogênio $=22,3 * * ;$ F/Plantas por cova $=17,2 * ;$ F/Interação $=2,9 *$; C.V. $=12,0 \%$. 
maneira geral, com o aumento dos níveis de N e quando o número de plantas por cova diminuiu de dois para um (Tabela 2). Esse aumento com a diminuição da densidade de plantio concorda com os resultados obtidos por Farias (1988) e Paris et al. (1988). Os maiores pesos ocorreram com 130 e $180 \mathrm{~kg} / \mathrm{ha}$ de $\mathrm{N}$, com uma planta por cova, e os menores com $0 \mathrm{~kg} / \mathrm{ha}$ de $\mathrm{N}$, em ambos casos de número de plantas por cova. Com uma planta por cova o peso com $130 \mathrm{~kg} / \mathrm{ha}$ de $\mathrm{N}$ foi maior do que com $80 \mathrm{~kg} / \mathrm{ha}$ de $\mathrm{N}$, enquanto com duas plantas por cova esse fato não se verificou.

Pela classificação do tamanho de frutos, constatou-se uma variação dos tipos 6 a 16, mas, a maioria dos frutos $(74,8 \%)$ concentrou-se nos tipos $8(23,5 \%)$, que é aceito pelo mercado interno e 10 e $12(51,3 \%)$, que são aceitos pelo mercado externo (Tabela 3 ). Entretanto, ocorreram $16,8 \%$ de frutos muito pequenos, tipos 14 e 16, principalmente onde havia duas plantas por cova, que são considerados frutos de qualidade inferior para os dois tipos de mercado.

O tratamento que apresentou a maior ocorrência $(55,7 \%)$ de frutos com tamanho mais indicado para o mercado interno, tipos 6 e 8 , foi o de $80 \mathrm{~kg} / \mathrm{ha}$ de $\mathrm{N}$, com uma planta por cova; e os tratamentos que apresentaram as maiores ocorrências $(60,8 \%$ e $61,7 \%)$ de frutos com tamanho mais indicado para o mercado externo, tipos 10 e 12, foram os de 130 e $180 \mathrm{~kg} / \mathrm{ha}$ de $\mathrm{N}$, respectivamente, com duas plantas por cova (Tabela 3), ilustrando que para o mercado

TABELA 2. Peso médio dos frutos de melão em função dos níveis de $\mathrm{N}$ e do número de plantas por cova. Juazeiro, BA, $1995^{1}$.

\begin{tabular}{|c|c|c|}
\hline \multirow{2}{*}{$\begin{array}{c}\text { Nitrogênio } \\
(\mathrm{kg} / \mathrm{ha})\end{array}$} & \multicolumn{2}{|c|}{ Número de plantas por cova } \\
\hline & 1 & 2 \\
\hline & \multicolumn{2}{|c|}{ 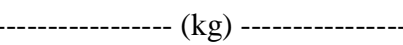 } \\
\hline 0 & $1,237 \mathrm{bA}$ & $1,008 \mathrm{bB}$ \\
\hline 80 & $1,389 \mathrm{bA}$ & $1,117 \mathrm{abA}$ \\
\hline 130 & $1,585 \mathrm{aA}$ & $1,232 \mathrm{aB}$ \\
\hline 180 & $1,705 \mathrm{aA}$ & $1,219 \mathrm{aB}$ \\
\hline
\end{tabular}

${ }^{1}$ Médias seguidas pela mesma letra minúscula nas colunas e maiúscula nas linhas não diferem pelo teste de Duncan a $5 \%$ de probabilidade; F/Nitrogênio $=32,2 * * ;$ F/Plantas por cova $=273,4 * ;$ F/Interação $=$ $3,4 * ;$ C.V. $=9,4 \%$ externo exige que se eleve mais o nível de $\mathrm{N}$ e a densidade de plantio do que para o mercado interno.

Observa-se que, da mesma forma que aconteceu para o peso médio de frutos, também a ocorrência de frutos de tamanhos maiores foi mais freqüente com uma planta por cova e, ao contrário, a ocorrência de frutos de tamanhos menores foi mais freqüente com duas plantas por cova (Tabelas 2 e 3), demonstrando uma relação direta entre peso médio e tamanho do fruto, como era de se esperar.

Pela Tabela 4, observa-se que o $\mathrm{N}$ exerceu um efeito positivo nos valores do Brix dos frutos do melão, o que está de acordo com os resultados de outros trabalhos (Srinivas \& Phabhakar, 1984; Faria et al., 1994).

TABELA 3. Porcentagem de frutos por nível de $\mathrm{Ne}$ número de plantas por cova, de acordo com a classificação por tamanho. Juazeiro, BA, 1995.

\begin{tabular}{|c|c|c|c|c|c|c|c|}
\hline \multirow{2}{*}{$\begin{array}{l}\text { Nitrogênio } \\
(\mathrm{kg} / \mathrm{ha})\end{array}$} & \multirow{2}{*}{$\begin{array}{c}\text { Planta/ } \\
\text { cova }\end{array}$} & \multicolumn{6}{|c|}{ Tipo (no de frutos/caixa) } \\
\hline & & 6 & 8 & 10 & 12 & 14 & 16 \\
\hline & & --- & ----- & ---- $\left(c^{c}\right.$ & \%) - & . & 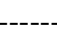 \\
\hline \multirow[t]{2}{*}{0} & 1 & 5,1 & 27,0 & 28,0 & 26,7 & 13,2 & 0,0 \\
\hline & 2 & 2,6 & 15,3 & 21,7 & 30,1 & 30,3 & 0,0 \\
\hline \multirow[t]{2}{*}{80} & 1 & 23,8 & 31,9 & 20,3 & 15,5 & 8,5 & 0,0 \\
\hline & 2 & 4,2 & 18,7 & 21,8 & 24,2 & 28,9 & 2,2 \\
\hline \multirow[t]{2}{*}{130} & 1 & 10,4 & 18,7 & 26,0 & 24,7 & 20,2 & 0,0 \\
\hline & 2 & 3,3 & 15,3 & 22,7 & 38,1 & 17,4 & 3,2 \\
\hline \multirow[t]{2}{*}{180} & 1 & 14,9 & 33,5 & 32,6 & 15,9 & 3,1 & 0,0 \\
\hline & 2 & 3,0 & 27,9 & 38,9 & 22,8 & 7,4 & 0,0 \\
\hline Média & & 8,4 & 23,5 & 26,6 & 24,7 & 16,1 & 0,7 \\
\hline
\end{tabular}

TABELA 4. Brix dos frutos de melão em função dos níveis de N. Juazeiro, BA, 1995'.

\begin{tabular}{cc}
\hline $\begin{array}{c}\text { Nitrogênio } \\
(\mathrm{kg} / \mathrm{ha})\end{array}$ & ${ }^{\circ}$ Brix \\
\hline 0 & $9,01 \mathrm{a}$ \\
80 & $10,22 \mathrm{~b}$ \\
130 & $10,29 \mathrm{~b}$ \\
180 & $10,61 \mathrm{~b}$ \\
\hline
\end{tabular}

${ }^{1}$ Médias seguidas pela mesma letra não diferem pelo teste de Duncan a $5 \%$ de probabilidade. 


\section{CONCLUSÕES}

1. Em relação aos níveis de $\mathrm{N}, 80 \mathrm{~kg} / \mathrm{ha}$ atinge o plateau de produtividade; o plateau de produtividade com duas plantas por cova é inferior ao de uma planta por cova.

2. Não há diferença significativa entre os espaçamentos de 1,80 e 2,00 m entre linhas nas características estudadas.

3. O peso médio de frutos do melão aumenta à medida que se aumenta os níveis de nitrogênio ou se diminui a densidade de plantio de duas para uma planta por cova.

4. Com o nível de $80 \mathrm{~kg} / \mathrm{ha}$ de $\mathrm{N}$, associado à densidade de uma planta por cova, é possível obter produtividade elevada, com boa parte de frutos próprios para o mercado interno.

5. Para obter uma maior parte de frutos próprios para o mercado externo, exige-se que se eleve a densidade para duas plantas por cova e se aumente o nível de $\mathrm{N}$ para 130 ou $180 \mathrm{~kg} / \mathrm{ha}$.

6. Com o nível de $80 \mathrm{~kg} / \mathrm{ha}$ de $\mathrm{N}$, também obtém-se frutos com 10,22 ${ }^{\circ}$ Brix, superior ao do tratamento sem $\mathrm{N}$ e não inferior aos dos outros níveis.

\section{REFERÊNCIAS}

BHELLA, H.S.; WILCOX, G.E. Yield composition of muksmelon as influenced by preplant and trickle applied nitrogen. Hortscience, Alexandria, VA, v.21, n.1, p.86-88, 1986.

BLACK, C.A. Soil plant relationships. New York : John Wiley \& Sons, 1968. 792p.

COSTA, E.F. da; FRANÇA, G.E. de; ALVES, V.M.C. Aplicação de fertilizantes via água de irrigação. Informe Agropecuário, Belo Horizonte, v.12, n.139, p.63- 69, 1986.

EMBRAPA. Serviço Nacional de Levantamento e Conservação de Solos (Rio de Janeiro, RJ). Manual de métodos de análises de solo. Rio de Janeiro, 1979. 1v.
FARIA, C.M.B. de. Nutrição mineral e adubação da cultura do melão. Petrolina, $\mathrm{PE}$ : EmbrapaCPATSA, 1990. 26p. (Embrapa-CPATSA. Circular técnica, 22).

FARIA, C.M.B. de; PEREIRA, J.R.; POSSÍDIO, E.L. Adubação orgânica e mineral na cultura do melão em um Vertissolo do Submédio São Francisco. Pesquisa Agropecuária Brasileira, Brasília, v.29, n.2, p.191-197,1994.

FARIAS, J.R.B. Comportamento da cultura do melão em estufa plástica, sob diferentes níveis de espaçamento, raleio e cobertura do solo. Pelotas : Universidade Federal de Pelotas, 1988. 80p. Dissertação de Mestrado.

JANICK, J. A Ciência de horticultura. 2.ed. Rio de Janeiro : Freitas Bastos, 1968. 485p.

MEISHERI, T.G.; JADAV, K.V.; PATEL, J.J.; PATEL, D.P. Effect of different levels of nitrogen and phosphorus on the fruit yield to muskmelon (Cucumis melo) var. GMM - 1. Guajarat Agricultural University Research Journal, v.9, n.2, p.10-13, 1984.

PARIS, H.S.; NERSON, H.; BURGER, Y.; EDELSTEIN, M.; KARCHI, Z. Synchrony of yield of melons as affected by plant type and density. Journal of Horticultural Science, Ashford, v.63, n.1, p.141-147, 1988.

PRABHAKAR, B.S.; SRINIVAS, K.; SHUKLA, V. Yield and quality of muskmelon (cv. Hara madhu) in relation to spacing and fertilization. Progressive Horticulture, v.17, n.1, p.51-55, 1985.

SRINIVAS, K.; PRABHAKAR, B.S. Response of muskmelon (Cucumis melo L.) to varying levels of spacing and fertilizers. Singapore Journal of Primary Industries, v.12, n.1, p.56-61, 1984.

WILCOX, G. E. Muskmelon response to rates and sources of nitrogen. Agronomy Journal, Madison, v.65, n.5, p.694-697, 1973. 